\title{
Structures and properties of molybdenum carbonyl complexes containing uninegative nitrogen-tripod ligands derived from heterocyclic compounds including 1-H-pyrazole and 1-H-1,2,4-triazole
}

\author{
Kom-Bei Shiu and Jean Y. Lee \\ Department of Chemistry, National Cheng Kung University, Tainan 70101 (Taiwan) \\ Yu Wang and Ming-Chu Cheng \\ Department of Chemistry, National Taiwan University, Taipei 10764 (Taiwan) \\ Sue-Lein Wang and Fen-Ling Liao \\ Department of Chemistry, National Tsing Hua University, Hsinchu 10764 (Taiwan) \\ (Received October 31, 1992; in revised form December 11, 1992)
}

\begin{abstract}
The melt reaction between 1-H-1,2,4-triazole $(\mathrm{HTz})$ and $\mathrm{NaBPh}_{4}$ affords $\mathrm{Na}\left(\mathrm{PhBTz}_{7}\right)$ while a similar process between $\mathrm{HTz}$ and $\mathrm{KBH}_{4}$ gives $\mathrm{K}\left(\mathrm{HBTz}_{3}\right)$. The uninegative $\mathrm{L}$ anion $\left(\mathrm{L}=\mathrm{PhBT}_{3}\right.$ or $\left.\mathrm{HBTz}_{3}\right)$ reacts with $\left[\mathrm{Mo}(\mathrm{CO})_{6}\right]$ to give $\left[\mathrm{MoL}(\mathrm{CO})_{3}\right]^{-}$. Upon addition of electrophiles, [MoL(CO $\left.)_{2}\left(\eta^{3} \text {-allyl)], [MoL(CO) }\right)_{2}(\mathrm{NO})\right],\left[\mathrm{MoL}(\mathrm{CO})_{3} \mathrm{Br}\right]$, and $\left[\mathrm{MoL}(\mathrm{CO})_{3} \mathrm{I}\right]$ are obtained. Two structures have been characterized by $\mathrm{X}$ ray crystallography: $\left[\mathrm{NEt}_{4}\right]\left[\mathrm{Mo}\left(\mathrm{HBPz}_{3}\right)(\mathrm{CO})_{3}\right]$, orthorhombic, $P b c a, a=15.832(3), b=15.799(4)$, $c=19.388(5) \AA, Z=8, R=0.043, R_{\mathrm{w}}=0.031$ based on 2591 reflections with $I>3.0 \sigma(I) ;\left[\mathrm{N}\left(\mathrm{PPh}_{3}\right)_{2}\right]\left[\mathrm{Mo}(\mathrm{HBTz})(\mathrm{CO})_{3}\right]$, orthorhombic, Pbca, $a=17.815(3), b=32.017(2), c=15.331(3) \AA, Z=8, R=0.044, R_{\mathrm{w}}=0.061$ based on 5743 reflections with $I>2.0 \sigma(I)$. Although comparison of the averaged $\nu(\mathrm{CO})$ values reveals the increasing order of the electron donativity as $\mathrm{HBBz}_{3}{ }^{-}<\mathrm{HBTz}_{3}{ }^{-}, \mathrm{PhBTz}_{3}{ }^{-}<\mathrm{HBPz}_{3}{ }^{-}<\mathrm{HBPz}_{3}^{\prime-}, \mathrm{HBPz}_{3}^{\star-}<\mathrm{HBPz}_{3}^{\prime \prime}$, neither structural features nor reactivities show an appreciable substituent effect (i.e., $\mathrm{HBTz}_{3}{ }^{-}$is. $\mathrm{HBPz}_{3}{ }^{-}$and $\mathrm{HBTz}_{3}{ }^{-}$is. $\mathrm{PhBTz}_{3}{ }^{-}$). Neither is the 5-methyl-group effect for nickel pseudohalides suggested by Trofimenko et al. supported strongly by the solid-state structures of $\left[\mathrm{MoL}^{\prime}(\mathrm{CO})_{3}\right]^{-}\left(\mathrm{L}^{\prime}=\mathrm{HBPz}_{3}{ }^{-}\right.$, $\mathrm{HBPz}_{3}^{\prime-}$, or $\mathrm{HBPz}_{3}^{\star--} ; \mathrm{Pz}^{\prime}=3,5$-dimethylpyrazol-1-yl; $\mathrm{Pz}^{\star}=3$-methylpyrazol-1-yl).
\end{abstract}

\section{Introduction}

Poly(pyrazolyl)borates have heen used extensively as multidentate ligands in the syntheses of numerous organometallic and coordination compounds, since they were introduced in the 1960s [1]. There has been renewed interest arising from novel reactions such as activation of small molecules like $\mathrm{H}_{2}$ [2], $\mathrm{O}_{2}$ [3] or $\mathrm{N}_{2}$ [4], induced by metal complexes without any carbonyl ligation, and of $\mathrm{C}-\mathrm{H}$ bonds observed for quite a few metal carbonyl derivatives containing the tripodal tris(pyrazolyl)borates [5]. We and others have prepared similar ligands and collected structural and spectral

Correspondence to: Dr. K.-B. Shiu. data [6] as well as carrying out orbital analysis [7] and determining relative formation rates [8] of the carbonyl-containing complexes of all these tridentate ligands. The aim has been to get a clear picture of the electronic or steric factors determining the observed reactivity. The recent report by Trofimenko et al. [9] on the importance of the methyl group on the pyrazolyl ring-5 position of tris(pyrazolyl)borates in nickel pseudohalides therefore prompted us to determine the crystal structures of a known compound, $\left[\mathrm{Et}_{4} \mathrm{~N}\right][\mathrm{Mo}-$ $\left.\left(\mathrm{HBPz}_{3}\right)(\mathrm{CO})_{3}\right][7 \mathrm{a}]$, and a new analogue, $\left[\mathrm{N}\left(\mathrm{PPh}_{3}\right)_{2}\right]$ $\left[\mathrm{Mo}\left(\mathrm{HBTz}_{3}\right)(\mathrm{CO})_{3}\right]$, and to study related reactions of this complex and another analogue, $\left[\mathrm{NEt}_{4}\right][\mathrm{Mo}(\mathrm{Ph}-$ $\left.\left.\mathrm{BTz}_{3}\right)(\mathrm{CO})_{3}\right]$, to understand if such an effect of 5methyl-group is also present in metal carbonyl deriva- 
tives containing the ligands, where $\mathrm{P}_{z}$ is pyrazol-1-yl and $\mathrm{Tz}$ is 1,2,4-triazol-1-yl.

\section{Experimental section}

All operations were performed by the usual Schlenk techniques [10], using doxygened, dry solvents and gases. IR spectra, calibrated with polystyrene, were recorded on a Hitachi Model 27()-30 instrument. Abbreviations are vs, very strong; $s$, strong; $m$, medium; $w$, weak; and sh, shoulder. NMR spectra were obtained on Bruker WP-100 ( $\left.{ }^{1} \mathrm{H}, 100 \mathrm{MHz}\right)$ or Varian VXR-300 $\left({ }^{1} \mathrm{H}, 300 \mathrm{MHz}\right)$ FT-NMR spectrometers. Chemical shifts $(\delta$ in ppm, $J$ in Herz) are defined as positive downfield or negative upfield relative to internal SiMe (TMS) standard. Abbreviations are s, singlet; d, doublet; br. unresolved multiplet or two overlapped singlets; tt, triplet of triplet; q, quartet. Elemental analyses were by the Microanalytical Service of the Department of Chemistry, National Cheng Kung University. [NEt $\left.{ }_{4}\right]\left[\mathrm{MO}\left(\mathrm{HBPz}_{3}\right)(\mathrm{CO})_{3}\right]$ was prepared by the established procedure [7a].

\subsection{Preparation of KHBTz;}

A mixture of 1-H-1,2,4-triazole (HTz, 29.29 g, 424 mmol) and $\mathrm{KBH}_{4}(5.72 \mathrm{~g}, 106 \mathrm{mmol})$ was heated slowly to $200^{\circ} \mathrm{C}$ until $7.81(318 \mathrm{mmol})$ of $\mathrm{H}_{2}$ had evolved (wet test meter). The melt was poured into $100 \mathrm{ml}$ of stirred tetrahydrofuran (THF) and the mixture cooled to room temperature. The white precipitate was collected and recrystallized from $\mathrm{MeOH} / \mathrm{Et}_{2} \mathrm{O}$ to give the product (14.87 g. 55\%). Anal. Found: C, 28.12: H. 2.87; N, 49.31. $\mathrm{C}_{6} \mathrm{H}_{7} \mathrm{BKN}_{9}$ calc.: $\mathrm{C}, 28.25 ; 11,2.77: \mathrm{N}, 49.42 \%$. IR (KBr): $v(\mathrm{BH}), 2484 \mathrm{w} \mathrm{cm}$ '. ' $\mathrm{H}$ NMR $25^{\circ} \mathrm{C}$. dimethylsulfoxide- $d_{6}, 100 \mathrm{MHz}$ ): protons at ring-3 and -5 positions, $\delta 8.13(3 \mathrm{H}, \mathrm{s}), 7.83(3 \mathrm{H}, \mathrm{s})$.

\subsection{Preparation of $\mathrm{NaPhBT} z_{;}$}

A mixture of $\mathrm{NaBPh}_{+}(13.00 \mathrm{~g}, 38 \mathrm{mmol})$ and $\mathrm{HTz}$ $(51.96 \mathrm{~g}, 753 \mathrm{mmol})$ was gradually heated to $250^{\circ} \mathrm{C}$ until ca. $8.1 \mathrm{~g}(104 \mathrm{mmol})$ of benzene had distilled from the melt. The mixture was then poured into 200 $\mathrm{ml}$ of THF and filtered immediately. The off-white solid residue was washed twice with $200 \mathrm{ml}$ of $\mathrm{THF}$ and once with $30 \mathrm{ml}$ of $\mathrm{MeOH}$. The solid was then recrystallized from $\mathrm{Me}_{2} \mathrm{SO}$ and air dried to give $10.7 \mathrm{~g}$ (89\%) product. Anal. Found: C, 45.54; H, 3.45; N, 39.87. $\mathrm{C}_{12} \mathrm{H}_{11} \mathrm{BN}_{9} \mathrm{Na}$ calc: $\mathrm{C}, 45.74 ; \mathrm{H}, 3.53 ; \mathrm{N}$, $40.01 \%$. ${ }^{2} \mathrm{H}$ NMR $\left(25^{\circ} \mathrm{C}\right.$, dimethylsulfoxide $-d_{0}, 100$ $\mathrm{MHz}$ ): protons at ring- 3 and -5 positions. $\delta 7.90(3 \mathrm{H}$. s), $7.78(3 \mathrm{H}, \mathrm{s})$; phenyl protons, $7.15(5 \mathrm{H}, \mathrm{m})$.

\subsection{Preparation of $/ \mathrm{NEt}_{4} / / \mathrm{Mo}\left(\mathrm{HBT} \mathrm{z}_{3}\right)(\mathrm{CO})_{3} /$}

A mixture of $1.98 \mathrm{~g}(7.7 \mathrm{mmol})$ of $\mathrm{KHBTz}_{3}$ and 1.85 $\mathrm{g}(7.0 \mathrm{mmol})$ of $\left[\mathrm{Mor}(\mathrm{CO})_{6}\right]$ in $50 \mathrm{ml}$ of $\mathrm{MeCN}$ was stirred at reflux for $3.5 \mathrm{~h}$ to give a yellow solution. A de-aerated solution of $2.1 \mathrm{~g}$ of $\mathrm{Et}_{+} \mathrm{NBr}$ in $150 \mathrm{ml}$ of $\mathrm{H}_{2} \mathrm{O}$ wats then added to the solution to give the crystalline solid. The pale yellow solid was collected and washed twice with $20 \mathrm{ml}$ of $\mathrm{H}, \mathrm{O}$ and three times with $10 \mathrm{ml}$ of $\mathrm{E}, \mathrm{O}$ and dried under vacuum; vield $3.00 \mathrm{~g}$ (83\%). Anal. Found: C, 38.6.3: H, 5.21: N. 26.41. $\mathrm{C}_{1} \mathrm{H}_{27} \mathrm{BMON}_{10} \mathrm{O}$, catc: $\mathrm{C}, 38.80: \mathrm{H}, 5.17 ; \mathrm{N} .26 .62 \%$. IR (KBr): $\nu(\mathrm{BH}) .252 \mathrm{iw} ; \nu(\mathrm{CO}), 1902 \mathrm{~s}, 1774 \mathrm{vs}$ and IR $(\mathrm{MeCN}): v(\mathrm{CO}), 1906 \mathrm{~s} .1776 \mathrm{vs} \mathrm{cm}$ ' $\mathrm{H}$ NMR $25^{\circ} \mathrm{C}$. acetone- $d_{0} .100 \mathrm{MHz}$ ): protons at ring-3 and -5 positions, $88.38(3 \mathrm{H}, 5), 8.13(3 \mathrm{H}, \mathrm{s}), \mathrm{CH}$, of $\mathrm{NE}_{4}, 3.47$ $(8 \mathrm{H}, \mathrm{q}, J(\mathrm{H} . \mathrm{H})=7.2) ; \mathrm{CH}_{3}$ of $\mathrm{NEt}_{4}, 1.36(12 \mathrm{H}, \mathrm{tt}$, $J(\mathrm{~N}, \mathrm{H})=1.9 . J(\mathrm{H}, \mathrm{H})=7.2)$. A metathesis reaction between bis(triphenylphosphine)ininium chloride (IN(P$\left.\mathrm{Ph}_{3},{ }_{2}, \mathrm{Cl}\right)$ and $\mathrm{K}\left[\mathrm{Mo}\left(\mathrm{HBTz}_{3}\right)(\mathrm{CO})_{3}\right]$ in $\mathrm{MeOH}$ was also found to give [N(PPh, $)_{2}$ [ [Mo(IIBT, $)\left(\mathrm{CO}_{3}\right]$.

\subsection{Preparation of / NEt //Mo(PhBT: $)(C O)_{;} /$}

This yellow compound was obtained from $\mathrm{NaPh}$ $\mathrm{BT} z_{3}$. [Mor(CO) $)_{4}$. and excess $\mathrm{Et}_{4} \mathrm{NBr}$ bv an analogous procedure; yield $80 \%$. Anal. Found: $\mathrm{C}, 40.21 \mathrm{H}, 5.46$; $\mathrm{N}, 23.49 . \mathrm{C}_{23} \mathrm{H}_{31} \mathrm{BMON}_{11} \mathrm{O}$; calc: $\mathrm{C}$. 45.86: H, 5.20: N, $23.25 \%$. IR (KBr): wCO). 1904s. 1770) and IR (MeCN): $\because(\mathrm{CO}) .1000 \mathrm{~s}, 1774 \mathrm{vs} \mathrm{cm}$ ' 'H NMR $25^{\circ} \mathrm{C}$. $\mathrm{CDCl}_{3}, 100 \mathrm{MHz}$ : protons at ring -3 and -5 positions, if $8.29(3 \mathrm{H}, s) .8 .09(3 \mathrm{H}, s)$ Ph. $7.86(2 \mathrm{H}, \mathrm{m}) .7 .56(3 \mathrm{H}$. m): $\mathrm{CH}_{2}$ of $\mathrm{NEt}, \ldots 3.33(8 \mathrm{H}, \mathrm{q}, J(\mathrm{H}, \mathrm{H})=7.3): \mathrm{CH}_{3}$ of $\mathrm{NEt}_{4}, 1.38(12 \mathrm{H}, 11, J(\mathrm{~N} . \mathrm{H})=1.9, J(\mathrm{H} . \mathrm{H})=7.3)$.

\subsection{Preparation of /Moll(CO), NO)/ $(L=H B T=$; PhBTz:)}

To a stirred solution of $3.0 \mathrm{mmol}$ of $\mathrm{M}\left[\mathrm{MoL}(\mathrm{CO})_{3}\right]$ $(\mathrm{M}=\mathrm{Na}$ or $\mathrm{K})$ prepared in sitt in $30 \mathrm{ml}$ of $\mathrm{MeCN}$. $[\mathrm{NO}]\left[\mathrm{BF}_{4}\right](0.38 \mathrm{~g} .3 .3 \mathrm{mmol})$ wats added in portions. Gas evolved briskly and when this stopped the solvent was removed under vacuum. Recrystallization from $\mathrm{MeOH} / \mathrm{H}_{2} \mathrm{O}$ gave the orange vellow product [Mo(HB-

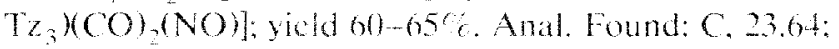
$\mathrm{H}, 1.93 ; \mathrm{N}$. 34.37. $\mathrm{C}_{\mathrm{H}} \mathrm{H}_{7} \mathrm{BMON}_{11} \mathrm{O}_{2} \cdot 1 / 2 \mathrm{H}_{2} \mathrm{O}$ calc.: $\mathrm{C}$. $23.70 ; H$ H. 1.95: N. 34.60\%. IR $(\mathrm{KBr}):,(\mathrm{BH}), 2544 \mathrm{w}$; ${ }^{2}(\mathrm{CO}), 2028 \mathrm{~s}, 1936 \mathrm{~s}, v(\mathrm{NO}) .1680 \mathrm{~s}$ and IR $(\mathrm{CH}, \mathrm{Cl}$, $\nu(\mathrm{CO}), 2036 \mathrm{~s}, 1950 \mathrm{~s} ;,(\mathrm{NO}), 1090 \mathrm{~s} \mathrm{~cm}^{-1},{ }^{1} \mathrm{H}$ NMR $\left(25^{\circ} \mathrm{C}\right.$. acetone $\left.-d_{1,}, 100 \mathrm{MHz}\right)$ : protons at ring -3 and -5 positions, $\delta 8.67(2 \mathrm{H}, s), 8.63(2 \mathrm{H}, \mathrm{s}), 8.58(1 \mathrm{H}, \mathrm{s}) .8 .54$ (1H. s). [Mo(PhBTz; $(\mathrm{CO}),(\mathrm{NO})]$. Anal. Found: $C$, 35,04; $\mathrm{H}, 2.49: \mathrm{N}, 29,13 . \mathrm{C}_{14} \mathrm{H}_{11} \mathrm{BMON}_{11} \mathrm{O}_{2} \cdot 1 / 2 \mathrm{H}_{2} \mathrm{O}$ calc.: $\mathrm{C}, 34.81 ; \mathrm{H}, 2.50:$ N. $29.00 \%$. IR $(\mathrm{KBr}): "(\mathrm{CO})$, 2028s. 1932s, $w(\mathrm{NO}), 1080 \mathrm{~s}$ and IR $\left(\mathrm{CH}_{2} \mathrm{Cl}_{2}\right):,(\mathrm{CO})$, $2036 \mathrm{~s}, 1948 \mathrm{~s} ; \times(\mathrm{NO}), 1680 \mathrm{~s} \mathrm{~cm}$ 'H NMR $25 \mathrm{C}$, $\mathrm{CDCl}_{3}, 300 \mathrm{MHz}$ ) protons at ring- 3 and -5 positions, $\delta$ $8.29(2 \mathrm{H}$ s) $8.27(2 \mathrm{H}$ s) $8.07(1 \mathrm{H}, \mathrm{s}) 8.00(1 \mathrm{H}, \mathrm{s}) ; \mathrm{Ph}$. $7.82(2 \mathrm{H}, \mathrm{m}), 7.58(3 \mathrm{H}, \mathrm{m})$. $\mathrm{Cl}(\mathrm{H})$ NMR $\left(25^{\circ} \mathrm{C}, \mathrm{CDCl}_{3}\right.$, $75 \mathrm{MHz}):(\mathrm{O}, 2210(2 \mathrm{C})$ : Carbon nucles at ring-3 and 
-5 positions, 155.4 (2 C), 155.2 (1 C), 149.7 (1 C), 149.5 (2 C); $\mathrm{Ph}, 133.8(2 \mathrm{C}), 130.3(1 \mathrm{C}), 129.4$ (2 C) with one unobserved signal for the ipso-carbon nucleus of $\mathrm{Ph}$.

\subsection{Preparation of $\left[\mathrm{MoL}(\mathrm{CO})_{3} X\right](X=\mathrm{Br}, \mathrm{I})$}

A solution of $3.03 \mathrm{mmol}$ of $\mathrm{M}\left[\mathrm{MoL}(\mathrm{CO})_{3}\right]$ in $20 \mathrm{ml}$ of $\mathrm{MeOH}$ was treated dropwise with a solution containing $3.40 \mathrm{mmol}$ of $\mathrm{Br}_{2}$ or $\mathrm{I}_{2}$ in $5 \mathrm{ml}$ of $\mathrm{MeOH}$ at $-20^{\circ} \mathrm{C}$ with immediate formation of precipitate. The resulting suspension was stirred for $10 \mathrm{~min}$ and then filtered out. The product was washed twice with $15 \mathrm{ml}$ of $\mathrm{MeOH}$ and dried under vacuum in $50-75 \%$ yield. [Mo( $\left.\left(\mathrm{HBTz}_{3}\right)(\mathrm{CO})_{3} \mathrm{I}\right]$, orange-yellow. Anal. Found: C, $20.53 ; \mathrm{H}, 1.46 ; \mathrm{N}, 24.05 . \mathrm{C}_{9} \mathrm{H}_{7} \mathrm{BIMoN}_{9} \mathrm{O}_{3}$ calc.: $\mathrm{C}$, $20.67 ; \mathrm{H}, 1.35 ; \mathrm{N}, 24.11 \%$. IR (KBr): $\nu(\mathrm{BH}), 2548 \mathrm{w}$; $\nu(\mathrm{CO}), 2044 \mathrm{~s}, 1968 \mathrm{~s}, 1934 \mathrm{~s}$ and IR $\left(\mathrm{CH}_{2} \mathrm{Cl}_{2}\right): \nu(\mathrm{CO})$, 2044s, $1974 \mathrm{~s}, 1942 \mathrm{~s} \mathrm{~cm}^{-1} .{ }^{1} \mathrm{H}$ NMR $\left(25^{\circ} \mathrm{C}, \mathrm{CDCl}_{3}, 100\right.$ $\mathrm{MHz}$ ): protons at ring- 3 and -5 positions, $\delta 8.74$ $(3 \mathrm{H}, \mathrm{s}), 8.36(3 \mathrm{H}, \mathrm{s})$. [Mo( $\left.\left.\mathrm{PhBTz}_{3}\right)(\mathrm{CO})_{3} \mathrm{Br}\right]$, yellowbrown. Anal. Found: $\mathrm{C}, 32.50 ; \mathrm{H}, 2.15 ; \mathrm{N}, 22.74$. $\mathrm{C}_{15} \mathrm{H}_{11} \mathrm{BBrMoN}_{9} \mathrm{O}_{3}$ calc.: $\mathrm{C}, 32.64 ; \mathrm{H}, 2.01 ; \mathrm{N}, 22.84 \%$. IR (KBr): $\nu(\mathrm{CO}), 2056 \mathrm{~s}, 1996 \mathrm{~s}, 1942 \mathrm{~s}$ and IR $\left(\mathrm{CH}_{2} \mathrm{Cl}_{2}\right)$ : $\nu(\mathrm{CO}), 2056 \mathrm{~s}, 1984 \mathrm{~s}, 1942 \mathrm{~s} \mathrm{~cm}^{-1} .{ }^{1} \mathrm{H}$ NMR $\left(25^{\circ} \mathrm{C}\right.$,
$\mathrm{CDCl}_{3}, 100 \mathrm{MHz}$ ): protons at ring-3 and -5 positions, $\delta$ $8.73(3 \mathrm{H}, \mathrm{s}), 8.62(3 \mathrm{H}, \mathrm{s}) ; \mathrm{Ph}, 7.86(2 \mathrm{H}, \mathrm{m}), 7.66(3 \mathrm{H}$, m). [ $\mathrm{Mo}\left(\mathrm{PhBTz}_{3}\right)(\mathrm{CO})_{3} \mathrm{I}$ ], orange-yellow. Anal. Found: $\mathrm{C}, 30.21 ; \mathrm{H}, 1.98 ; \mathrm{N}, 21.15 . \mathrm{C}_{15} \mathrm{H}_{11} \mathrm{BIMoN}_{9} \mathrm{O}_{3}$ calc.: $\mathrm{C}$, $30.08 ; \mathrm{H}, 1.85 ; \mathrm{N}, 21.05 \%$. IR (KBr): $\nu(\mathrm{CO}), 2036 \mathrm{~s}$, $1974 \mathrm{~s}, 1948 \mathrm{~s}$ and IR $\left(\mathrm{CH}_{2} \mathrm{Cl}_{2}\right): \nu(\mathrm{CO}), 2044 \mathrm{~s}, 1974 \mathrm{~s}$, $1940 \mathrm{~s} \mathrm{~cm}^{-1}$. ${ }^{1} \mathrm{H}$ NMR $\left(25^{\circ} \mathrm{C}, \mathrm{CDCl}_{3}, 100 \mathrm{MHz}\right)$ : protons at ring- 3 and -5 positions, $\delta 8.37(2 \mathrm{H}, \mathrm{s}) ; \mathrm{Ph}, 7.86$ $(2 \mathrm{H}, \mathrm{m}), 7.65(3 \mathrm{H}, \mathrm{m})$.

\subsection{Preparation of $\left[\mathrm{MoL}(\mathrm{CO})_{3}\left(\eta^{3}\right.\right.$-allyl $\left.)\right]$}

To a suspension of $1.0 \mathrm{mmol}$ of $\left[\mathrm{Et}_{4} \mathrm{~N}\right]\left[\mathrm{MoL}(\mathrm{CO})_{3}\right]$ in $20 \mathrm{ml}$ of $\mathrm{CH}_{2} \mathrm{Cl}_{2}$ was added $1 \mathrm{ml}$ of allyl bromide. The mixture was stirred at room temperature for $3 \mathrm{~h}$ and a transparent solution formed. A solution IR spectrum indicated the complete reaction. The solvent and excess allyl bromide were then removed under vacuum. Recrystallization from $\mathrm{CH}_{2} \mathrm{Cl}_{2} / \mathrm{MeOH}$ gave $80 \%$ of the orange-yellow solid. [ $\mathrm{Mo}\left(\mathrm{HBTz}_{3}\right)(\mathrm{CO})_{2}\left(\eta^{3}\right.$-allyl $\left.)\right]$, yellow. Anal. Found: C, 31.52; H, 3.04; N, 29.87. $\mathrm{C}_{11} \mathrm{H}_{12} \mathrm{BMoN}_{9} \mathrm{O}_{2} \cdot 1 / 2 \mathrm{H}_{2} \mathrm{O}$ calc.: $\mathrm{C}, 31.60 ; \mathrm{H}, 3.14$; $\mathrm{N}, 30.15 \%$. IR (KBr): $\nu(\mathrm{CO}), 1954 \mathrm{~s}, 1856 \mathrm{~s}$ and IR $\left(\mathrm{CH}_{2} \mathrm{Cl}_{2}\right): \nu(\mathrm{CO}), 1960 \mathrm{~s}, 1866 \mathrm{~s} \mathrm{~cm} \mathrm{~cm}^{-1} \cdot{ }^{1} \mathrm{H}$ NMR $\left(25^{\circ} \mathrm{C}\right.$,

TABLE 1. Crystal dala for $\left[\mathrm{NEl}_{4}\right]\left[\mathrm{Mu}\left(\mathrm{HBP}_{3}\right)(\mathrm{CO})_{3}\right]$ and $\left[\mathrm{N}\left(\mathrm{PPh}_{3}\right)_{2}\right]\left[\mathrm{Mo}\left(\mathrm{HBTz}_{3}\right)(\mathrm{CO})_{3}\right]$

\begin{tabular}{|c|c|c|}
\hline Compound & {$\left[\mathrm{NEt}_{4}\right]\left[\mathrm{Mo}\left(\mathrm{HBPz}_{3}\right)(\mathrm{CO})_{3}\right]$} & {$\left[\mathrm{N}\left(\mathrm{PPh}_{3}\right)_{2}\right]\left[\mathrm{Mo}\left(\mathrm{HBTz}_{3}\right)(\mathrm{CO})_{3}\right]$} \\
\hline $\begin{array}{l}\text { empirical formula } \\
\text { colour }\end{array}$ & $\begin{array}{l}\mathrm{C}_{20} \mathrm{H}_{30} \mathrm{BMoN}_{7} \mathrm{O}_{3} \\
\text { yellow }\end{array}$ & $\begin{array}{l}\mathrm{C}_{45} \mathrm{H}_{37} \mathrm{BMoN}_{10} \mathrm{O}_{3} \mathrm{P}_{2} \\
\text { yellow }\end{array}$ \\
\hline crystal size $(\mathrm{mm})$ & $0.60 \times 0.40 \times 0.34$ & $0.30 \times 0.40 \times 0.50$ \\
\hline space group & \multicolumn{2}{|c|}{ orthorhombic, $P b c a($ No. 61) } \\
\hline unit cell dimensions $a, b, c, \AA$ & $15.832(3), 15.799(4), 19.388(5)$ & $17.815(3), 32.017(2), 15.331(3)$ \\
\hline volume, $\AA^{3}$ & $4850(2)$ & $8745(2)$ \\
\hline$Z$ & 8 & 8 \\
\hline$D_{\text {calc }} \mathrm{g} / \mathrm{cm}^{3}$ & 1.433 & 1.336 \\
\hline orientation rflns, range & $13,13^{\circ} \leq 2 \theta \leq 24^{\circ}$ & $25,79^{\circ} \leq 2 \theta \leq 80^{\circ}$ \\
\hline data collected & $-h,-k,-l$ & $+h,+k,+l$ \\
\hline abs cor & $\psi$ scan & \\
\hline abs coeff, $\mathrm{mm}^{-1}$ & 0.56 & 3.62 \\
\hline abs. correction & not applied & applied \\
\hline transm range & - & $0.51-1.00$ \\
\hline diffractometer used & Siemens $\mathrm{R} 3 \mathrm{~m} / \mathrm{V}$ & Rigaku AFC-5R \\
\hline radiation; $\lambda, \AA$ & Mo $\mathrm{K} \alpha, 0.7107$ & $\mathrm{Cu} \mathrm{K} \alpha, 1.5418$ \\
\hline temperature $(\mathrm{K})$ & 297 & 298 \\
\hline scan type & $\theta / 2 \theta$ & \\
\hline $2 \theta$ range, deg & $3-50$ & $2-120$ \\
\hline scan speed, deg/min & $3-15$ & 32 \\
\hline std rflns & $3 \mathrm{std} / 50$ rflns & $3 \mathrm{std} / 300 \mathrm{rflns}$ \\
\hline decay; \% & $\leq 1$ & $\leq 1$ \\
\hline no. of unique rflns & 4284 & 6492 \\
\hline no. of rflns used & 2591 with $I>3.0 \sigma(I)$ & 5743 with $I>2.0 \sigma(I)$ \\
\hline $\max \Delta / \sigma$ ratio & 0.001 & 0.024 \\
\hline$R R_{\omega} \mathrm{S}^{\mathrm{a}}$ & $0.043,0.031,1.0$ & $0.044,0.061,2.45$ \\
\hline resid peak; e $/ \AA^{3}$ & 0.88 & 0.58 \\
\hline solution & direct method & Patterson synthesis \\
\hline
\end{tabular}

a See ref. [6a,11]. 
TABLE 2. Fractional atomic coordinates for [NF: $\left.{ }_{1}\right]\left[\mathrm{Mo}\left(\mathrm{HBP} z_{3}\right)\right.$ $\left(\mathrm{CO}_{3}\right]$ and $\left[\mathrm{N}\left(\mathrm{PPh}_{3}\right)_{2}\right]\left[\mathrm{Mo}\left(\mathrm{HBT}_{3}\right)(\mathrm{CO})_{3}\right]$

\begin{tabular}{|c|c|c|c|}
\hline Atom & $x$ & $y$ & $\ddot{z}$ \\
\hline \multicolumn{4}{|c|}{ (a) $/ \mathrm{NEt}_{+} / / \mathrm{MO}\left(\mathrm{HBPz} z_{i}\right)(\mathrm{CO})_{3} /$} \\
\hline Mo & $0.0605(1)$ & $0.1414(1)$ & $0.66609(1)$ \\
\hline$N$ & $0.7863(3)$ & $0.1205(2)$ & $0.0096(2)$ \\
\hline$N(11)$ & $0.046 .5(2)$ & $0.1837(2)$ & $0.5559(2)$ \\
\hline$N(12)$ & $0.083 .3(2)$ & $0.2571(2)$ & $0.5328(2)$ \\
\hline$N(21)$ & $0.1950(2)$ & $0.1809(2)$ & $0.64432)$ \\
\hline$N(22)$ & $0.2134(2)$ & $0.2520(2)$ & $0.607502)$ \\
\hline$N(31)$ & $0.0489(2)$ & $0.2832(2)$ & $0.6842(2)$ \\
\hline$N(32)$ & $0.0898(3)$ & $0.3397(2)$ & $0.6+20(2)$ \\
\hline$O(1)$ & $0.0792(3)$ & $0.1091(3)$ & $0.824 .3(2)$ \\
\hline$O(2)$ & $-0.1246(3)$ & $0.0848(3)$ & $0.60) 32(2)$ \\
\hline$O(3)$ & $0.0947(3)$ & $-0.0479(2)$ & $10.6348(2)$ \\
\hline B & $0.1415(3)$ & $0.3090(3)$ & 0.5813033 \\
\hline$(C 1)$ & $0.10745(3)$ & $0.1201(3)$ & $0.7640(2)$ \\
\hline$(x)$ & $-0.0552(3)$ & $0.1(093(3)$ & $0.68823(2)$ \\
\hline$C(3)$ & $0.0820(3)$ & $0.0241(3)$ & $0.6469(2)$ \\
\hline$C(4)$ & $0.8502(4)$ & $0.0511(4)$ & $0.1075(3)$ \\
\hline$C(5)$ & $0.7335(4)$ & $0.108+3)$ & $0.0344(3)$ \\
\hline$C(6)$ & $0.7279(4)$ & $0.1214(5)$ & $0.1603(3)$ \\
\hline$(x)$ & $0.8354(4)$ & $0.2032(4)$ & $(1.19910(3)$ \\
\hline$C(8)$ & $0.9091(5)$ & $0.0375(5)$ & $0.0481(4)$ \\
\hline$C(9)$ & $0.6918(4)$ & $0.0238(3)$ & $(1.11277(3)$ \\
\hline$C(10)$ & $0.7702(5)$ & $0.1398(8)$ & $0.2295(3)$ \\
\hline$(111)$ & $0.783 .3(5)$ & $0.2 \times \geq 0(4)$ & $0.118 \times 5(5)$ \\
\hline$C(13)$ & $0.0628(3)$ & $0.2698(.3)$ & $10 .+60.5(2)$ \\
\hline$C(14)$ & $0.01 .35(3)$ & $0.2039(3)$ & $10.4456(2)$ \\
\hline$C(15)$ & $0.0049(3)$ & $0.1520(3)$ & $0.50125(2)$ \\
\hline$C(23)$ & $0.2982(3)$ & $0.2617(3)$ & $0.603 .3 .3(3)$ \\
\hline$O(24)$ & $0.3349(3)$ & $0.1949(4)$ & $0.6 .37+1(3)$ \\
\hline$C(25)$ & $0.2697(3)$ & $0.1468(3)$ & $0.6022(3)$ \\
\hline$C(3,3)$ & $0.0794(3)$ & $0.4181(3)$ & $0.6690(3)$ \\
\hline$C(34)$ & $0.01317(4)$ & $01.4133(3)$ & $(1.7277(3)$ \\
\hline$(9.5)$ & $0.0135(3)$ & $0.3283(3)$ & $11,73+8(2)$ \\
\hline \multicolumn{4}{|c|}{ (b) $\left./ \mathrm{N}\left(P P h_{3}\right)_{2} / / \mathrm{Mo} / \mathrm{HBT} Z_{3}\right)(\mathrm{CO})_{3} /$} \\
\hline Mo & $0.768202(17)$ & $0.3558777(10)$ & $(1.225822(20)$ \\
\hline$O(1)$ & $0.85777(21)$ & $(1.29809(11)$ & $0.1094(3)$ \\
\hline$O(2)$ & $0.86708(18)$ & $0.31898(11)$ & $0.36471(21)$ \\
\hline$O(3)$ & $(0.89743(18)$ & $0.42294(12)$ & $10.220494(21)$ \\
\hline$C(1)$ & $0.82347(23)$ & $0.32118(13)$ & $10.152 \times(3)$ \\
\hline$C(2)$ & $(0.826 .53(22)$ & $0.33401(14)$ & $0.3179(3)$ \\
\hline$C(3)$ & $0.84902(21)$ & $0.3986 .3(14)$ & $01.220 .3(3)$ \\
\hline B & $0.5818(3)$ & $0.38246(16)$ & $0.20 .40(3)$ \\
\hline$N(11)$ & $0.68981(16)$ & $0.401 .4 .5(10)$ & $0.3(3353(20)$ \\
\hline$N(12)$ & $0.61598(17)$ & $0.40568(20)$ & $0.28172(20)$ \\
\hline$N(14)$ & $0.63386(19)$ & $0.44505(11)$ & $(0.39782(22)$ \\
\hline$N(21)$ & $0.7(0055(17)$ & $0.38732(10)$ & $0111510(20)$ \\
\hline $\mathrm{N}(22)$ & $0.62579(17)$ & $0.39500(10)$ & $(1.12201(21)$ \\
\hline$N(24)$ & $0.660076(22)$ & $(0.42104(1.3)$ & $-11.001427(23)$ \\
\hline$N(31)$ & $0.66240(19)$ & $0.3191 .5(10)$ & $0.23788(22)$ \\
\hline$N(32)$ & $0.593 .36(18)$ & $0.33530(11)$ & (i. $22(1) 64(21)$ \\
\hline$N(34)$ & $0.57576(24)$ & $0.26899(12)$ & $0.2532(3)$ \\
\hline$C(13)$ & $0.58521(22)$ & $0.43172(13)$ & $0.3400(3)$ \\
\hline$((15)$ & $0.69744(24)$ & $0.42556(12)$ & $0.372 \times(3)$ \\
\hline$C(23)$ & $0.60511(25)$ & $0.41487(14)$ & $(1.04960(3)$ \\
\hline$C(25)$ & $0.71823(25)$ & $0.40276(14)$ & $(1.013816 .3)$ \\
\hline$C(33)$ & $0.5437(3)$ & $0.30458(17)$ & $0.2301(3)$ \\
\hline$C(35)$ & $0.6486(3)$ & $0.28004(15)$ & $0.2573(3)$ \\
\hline$N$ & $0.57170(18)$ & $0.37501(11)$ & $(0.77408(19)$ \\
\hline $\mathrm{P}(1)$ & $0.50041(5)$ & $0.3576+13)$ & $0.8226946)$ \\
\hline $\mathrm{P}(2)$ & $0.59836(5)$ & $0.390 / 4(3)$ & $0.08135(6)$ \\
\hline
\end{tabular}

TABIE 2 (continued)

\begin{tabular}{|c|c|c|c|}
\hline Alom & l & $y$ & $=$ \\
\hline$(Y 11)$ & $0.1266,5(2))$ & $1134011(12)$ & $0.75135(25)$ \\
\hline$C(12 \mathrm{~A})$ & $11.36688+(24)$ & $(0.36588(1.3)$ & $0.7303(3)$ \\
\hline$((1,3)$ & 1131550124 & $0.35315(16)$ & $0.6688(3)$ \\
\hline$(C)+A)$ & $(1.2221(3)$ & $0.31,546(16)$ & $0.6297(3)$ \\
\hline$C(15 \mathrm{~A})$ & 0.380003 & $(1.25031115)$ & $0.65(13(3)$ \\
\hline$((1,1, A)$ & $01.436092+1$ & $(0.301,301(1.3)$ & $1.7097(3)$ \\
\hline$((3 \mid N)$ & $0.527820201)$ & $0.31596(11)$ & 1) $80428(23)$ \\
\hline$((22 A)$ & $(1.48(1)+(22)$ & $(0,28335(13)$ & 10.915009 \\
\hline$(23 A)$ & 0.5030433 & $(1,25358(14)$ & $0.9749(3)$ \\
\hline$(124 A)$ & $0.5723(3)$ & $(1.2505 .3(1.4)$ & $1.0159(3)$ \\
\hline$(125 \mathrm{~A})$ & $0.614+60(25)$ & $(1.28945(15)$ & $(1.0944(3)$ \\
\hline$(726 \wedge)$ & $0.5988(122)$ & 0.3187501 .31 & $11033603\}$ \\
\hline$(131)$ & 0.460750213 & $11.34757(12)$ & $11.8922(3)$ \\
\hline$(132 \mathrm{~A})$ & 11.42 .270231 & $10.38621011+1$ & $0.9678(.3)$ \\
\hline$(C 3 A)$ & 0.344503 & $(1 .+174+16)$ & 1.42013 \\
\hline$((34 A)$ & $0 .+113213$ & $(1.45417(1)$ & $0.9044(+)$ \\
\hline$(135 \mathrm{~A})$ & $0+41163$ & $(1.76404015)$ & $0.4253(4)$ \\
\hline$C(36 \mathrm{~A})$ & 0.47660 .31 & $(1.4 .392(11)+1$ & $0.8715(3)$ \\
\hline$(C(1 \perp B)$ & $0.66(179021)$ & $(1.433040111)$ & $0.69520(2.4)$ \\
\hline$(Y(12 B)$ & $0.6 \times 54(3)$ & $(1.44430(13)$ & $0.7776(3)$ \\
\hline$((13 B)$ & 0.7306031 & $(1.47630167)$ & $0.7 \times 72(3)$ \\
\hline$((1+B)$ & $0.704 .73)$ & $0.4070 .9418)$ & $0.715 .3(3)$ \\
\hline$((15 B)$ & $0.7379(3)$ & $11.4876,4(15)$ & $0.6337(3)$ \\
\hline$(16 \mathrm{~B})$ & $0.680 .303)$ & $11.45574(1.7)$ & $016232(3)$ \\
\hline$(C \mid B)$ & $0.52568020)$ & $0.41671112)$ & $0.6(17 \times 1(24)$ \\
\hline$(\triangle 2 \geq B)$ & $1150061(25)$ & $11+1760(18)$ & $0.60103(3)$ \\
\hline$((2.3 B)$ & $3.4+53.30$ & $0.40000(14)$ & $(0.5532(3)$ \\
\hline$(2413)$ & $0 .+1468(24)$ & $0.43302(15)$ & $(1.4446(3)$ \\
\hline$((25 B)$ & $(1.4 .8635(25)$ & $0.39220(14)$ & $0.49 \times 9(3)$ \\
\hline$(126 \mathrm{~B})$ & $(0.40257(22)$ & $0,27841(13)$ & $0.5496(3)$ \\
\hline$(73 \mid B)$ & $0.6540,3(21)$ & $11.35116(11)$ & $(0.026+1(25)$ \\
\hline$(1,3 \mathrm{~B})$ & 0.607789 & $01.35375(1.3)$ & $0.5372(3)$ \\
\hline$(C B B B)$ & $017172(3)$ & $11.32600(16)$ & (1). $49 \mathrm{Kon}(3)$ \\
\hline$(C(3+13)$ & $0.7545(3)$ & $0.20757(15)$ & $0.5472(3)$ \\
\hline$((3513)$ & $11.7118(25)$ & $0.2 y+31(14)$ & $0.6 .330(3)$ \\
\hline$((3 \cap B)$ & $(1.69001)(2.3)$ & $0.320186(12)$ & $0.6750(3)$ \\
\hline
\end{tabular}

$\left.\mathrm{CDCl}_{3}, 100 \mathrm{MHz}\right)$ : protons at ring -3 and -5 positions, $\delta$ $8.35(3 \mathrm{H}$, br) $8.22(3 \mathrm{H}$. br $)$; the central proton of allyl, $3.78(1 \mathrm{H}$. s); the sy-protons of allyl. $3.73(2 \mathrm{H}$, br $)$; the anti-protons of allyl, $1.66(2 \mathrm{H}$. d. $/(\mathrm{H}, \mathrm{H})=7.8)$. [Mo(PhBT $\left.z_{3}\right)(\mathrm{CO}),\left(\eta \eta^{3}\right.$-allyl $\left.)\right]$, yellow. Anal. Found: $\mathrm{C}$, 41.80; H, 3.35; N. 25.83, $\mathrm{C}_{17} \mathrm{H}_{16} \mathrm{BMON}_{4} \mathrm{O}$, calc.: $\mathrm{C}$. $42.09 \% \mathrm{H}, 3.32 ; \mathrm{N}, 25.99 \%$ IR $(\mathrm{KBr}):{ }_{1}(\mathrm{CO}), 1954 \mathrm{~s}$, $1856 \mathrm{~s}$, and IR $\left(\mathrm{CH}_{2} \mathrm{Cl}_{2}\right) ; \nu(\mathrm{CO}), 1960 \mathrm{~s}, 1866 \mathrm{~cm}^{-1}$. ${ }^{1} \mathrm{H}$ NMR $\left(25^{\circ} \mathrm{C} . \mathrm{CDCl}_{3}, 100 \mathrm{MH}\right)$ : protons at ring-3 and -5 positions, $\delta 8.39$ ( $3 \mathrm{H}$, br) $, 7.98(3 \mathrm{H}, \mathrm{br}): \mathrm{Ph}, 7.80$ $(2 \mathrm{H}, \mathrm{m}), 7.62(3 \mathrm{H}, \mathrm{m})$; the central proton of allyl. 3.87 $(1 \mathrm{H}, \mathrm{s})$ : the syn-protons of allyl. $3.76(2 \mathrm{H}$. br): the anti-protons of allyl. $1.67(2 \mathrm{H}$. d. $J(\mathrm{H}, \mathrm{H})=7.8)$.

2.8. X-Ray diffraction study of $/ \mathrm{NEt}_{4} / / \mathrm{Mo}\left(\mathrm{HBPz}_{3}\right)$ $\left(\mathrm{CO}_{3} /\right.$ and $\left./ \mathrm{N}\left(\mathrm{PP}_{3}\right)_{2}\right) / \mathrm{Mo}\left(\mathrm{HBT} \mathrm{z}_{3}\right)(\mathrm{CO})_{3} /$

All the single crystals were grown from $\mathrm{CH}_{2} \mathrm{Cl}_{2} /$ hexane at room temperature. General procedures and listings of programs were previously given $[6 a, 11]$. Absorption correction was performed on the structures 
TABLE 3. Selected bond lengths $(\AA)$ and angles $\left(^{\circ}\right)$ for $\left[\mathrm{NEt}_{4}\right]\left[\mathrm{Mo}\left(\mathrm{HBPz}_{3}\right)(\mathrm{CO})_{3}\right]$ and $\left[\mathrm{N}\left(\mathrm{PPh}_{3}\right)_{2}\right]\left[\mathrm{Mo}\left(\mathrm{HBTz}_{3}\right)(\mathrm{CO})_{3}\right]$

\begin{tabular}{|c|c|c|c|}
\hline \multicolumn{4}{|c|}{$\begin{array}{l}\text { (a) }\left[\mathrm{NEt}_{4}\right]\left[\mathrm{Mo}\left(\mathrm{HBPz}_{3}\right)(\mathrm{CO})_{3}\right] \\
\text { (i) Bond lengths }\end{array}$} \\
\hline $\mathrm{N}-\mathrm{C}(4)$ & $1.500(7)$ & $C(4)-C(8)$ & $1.497(9)$ \\
\hline $\mathrm{N}-\mathrm{C}(5)$ & $1.520(7)$ & $C(5)-C(y)$ & $1.498(8)$ \\
\hline $\mathrm{N}-\mathrm{C}(6)$ & $1.497(7)$ & $C(6)-C(10)$ & $1.527(9)$ \\
\hline \multirow[t]{2}{*}{$\mathrm{N}-\mathrm{C}(7)$} & $1.528(7)$ & $C(7)-C(11)$ & $1.495(9)$ \\
\hline & $n=1$ & $n=2$ & $n=3$ \\
\hline Mo-C $(n)$ & $1.926(4)$ & $1.924(5)$ & $1.925(5)$ \\
\hline Mo-N $(n 1)$ & $2.263(3)$ & $2.261(4)$ & $2.272(4)$ \\
\hline $\mathrm{B}-\mathrm{N}(n 2)$ & $1.551(6)$ & $1.537(6)$ & $1.527(6)$ \\
\hline $\mathrm{C}(n)-\mathrm{O}(n)$ & $1.167(6)$ & $1.184(7)$ & $1.177(6)$ \\
\hline $\mathrm{N}(n 1)-\mathrm{N}(n 2)$ & $1.373(5)$ & $1.362(5)$ & $1.362(5)$ \\
\hline $\mathrm{N}(n 2)-\mathrm{C}(n 3)$ & $1.341(6)$ & $1.355(6)$ & $1.348(6)$ \\
\hline $\mathrm{C}(n 3)-\mathrm{C}(n 4)$ & $1.363(7)$ & $1.373(8)$ & $1.365(8)$ \\
\hline $\mathrm{C}(n 4)-\mathrm{C}(n 5)$ & $1.382(7)$ & $1.370(7)$ & $1.381(7)$ \\
\hline $\mathrm{C}(n 5)-\mathrm{N}(n 1)$ & $1.326(6)$ & $1.346(6)$ & $1.337(6)$ \\
\hline \multicolumn{4}{|l|}{ (ii) Bond angles } \\
\hline $\mathrm{C}(4)-\mathrm{N}-\mathrm{C}(5)$ & $111.3(4)$ & $C(6)-N-C(7)$ & $112.7(5)$ \\
\hline $\mathrm{C}(4)-\mathrm{N}-\mathrm{C}(6)$ & $110.1(4)$ & $\mathrm{N}-\mathrm{C}(4)-\mathrm{C}(8)$ & $116.5(5)$ \\
\hline $\mathrm{C}(4)-\mathrm{N}-\mathrm{C}(7)$ & $107.0(4)$ & $\mathrm{N}-\mathrm{C}(5)-\mathrm{C}(9)$ & $115.6(5)$ \\
\hline$C(5)-N-C(6)$ & $108.0(4)$ & $\mathrm{N}-\mathrm{C}(6)-\mathrm{C}(10)$ & $114.9(5)$ \\
\hline$C(5)-N-C(7)$ & $107.7(4)$ & $\mathrm{N}-\mathrm{C}(7)-\mathrm{C}(11)$ & $115.8(5)$ \\
\hline $\mathrm{C}(1)-\mathrm{Mo}-\mathrm{C}(2)$ & $84.9(2)$ & $\mathrm{C}(1)-\mathrm{Mo}-\mathrm{N}(11)$ & $172.9(2)$ \\
\hline $\mathrm{C}(1)-\mathrm{Mo}-\mathrm{C}(3)$ & $90.4(2)$ & $\mathrm{C}(1)-\mathrm{Mo}-\mathrm{N}(21)$ & $97.4(2)$ \\
\hline $\mathrm{C}(2)-\mathrm{Mo}-\mathrm{C}(3)$ & $86.8(2)$ & $\mathrm{C}(1)-\mathrm{Mo}-\mathrm{N}(31)$ & $92.1(1)$ \\
\hline $\mathrm{N}(11)-\mathrm{Mo}-\mathrm{N}(21)$ & $80.0(1)$ & $\mathrm{C}(2)-\mathrm{Mo}-\mathrm{N}(11)$ & $97.6(2)$ \\
\hline $\mathrm{N}(11)-\mathrm{Mo}-\mathrm{N}(31)$ & $80.9(1)$ & $\mathrm{C}(2)-\mathrm{Mo}-\mathrm{N}(21)$ & $117.6(2)$ \\
\hline $\mathrm{N}(21)-\mathrm{Mo}-\mathrm{N}(31)$ & $80.4(1)$ & $\mathrm{C}(2)-\mathrm{Mo}-\mathrm{N}(31)$ & $99.2(2)$ \\
\hline $\mathrm{N}(12)-\mathrm{B}-\mathrm{N}(22)$ & $109.3(4)$ & $\mathrm{C}(3)-\mathrm{Mo}-\mathrm{N}(11)$ & $96.4(2)$ \\
\hline$N(12)-B-N(32)$ & $108.9(4)$ & $\mathrm{C}(3)-\mathrm{Mo}-\mathrm{N}(21)$ & $93.5(2)$ \\
\hline \multirow[t]{2}{*}{$N(22)-B-N(32)$} & $109.0(4)$ & $\mathrm{C}(3)-\mathrm{Mo}-\mathrm{N}(31)$ & $173.6(2)$ \\
\hline & $n=1$ & $n=2$ & $n=3$ \\
\hline $\mathrm{Mo}-\mathrm{N}(n 1)-\mathrm{N}(n 2)$ & $121.2(3)$ & $122.0(3)$ & $121.4(3)$ \\
\hline $\mathrm{Mo}-\mathrm{N}(n 1)-\mathrm{C}(n 5)$ & $132.8(3)$ & $131.8(3)$ & $131.9(3)$ \\
\hline $\mathrm{Mo}-\mathrm{C}(n)-\mathrm{O}(n)$ & $176.7(5)$ & $176.0(4)$ & $179.7(5)$ \\
\hline $\mathrm{B}-\mathrm{N}(n 2)-\mathrm{N}(n 1)$ & $120.0(3)$ & $119.9(4)$ & $120.4(3)$ \\
\hline $\mathrm{B}-\mathrm{N}(n 2)-\mathrm{C}(n 3)$ & $130.2(4)$ & $130.4(4)$ & $130.4(4)$ \\
\hline $\mathrm{N}(n 1)-\mathrm{N}(n 2)-\mathrm{C}(n 3)$ & $109.7(4)$ & $109.6(4)$ & $109.1(4)$ \\
\hline $\mathrm{N}(n 2)-\mathrm{C}(n 3)-\mathrm{C}(n 4)$ & $108.0(4)$ & $107.7(5)$ & $108.9(4)$ \\
\hline $\mathrm{C}(n 3)-\mathrm{C}(n 4)-\mathrm{C}(n 5)$ & $105.8(4)$ & $106.1(5)$ & $105.0(5)$ \\
\hline $\mathrm{C}(n 4)-\mathrm{C}(n 5)-\mathrm{N}(n 1)$ & $110.6(4)$ & $110.4(5)$ & $110.6(4)$ \\
\hline $\mathrm{N}(n 2)-\mathrm{N}(n 1)-\mathrm{C}(n 5)$ & $106.0(3)$ & $106.2(4)$ & $106.4(4)$ \\
\hline \multicolumn{4}{|c|}{$\begin{array}{l}\text { (b) [N }\left(\mathrm{PPh}_{3}\right)_{2} /\left[\mathrm{Mo}\left(\mathrm{HBTz}_{3}\right)(\mathrm{CO})_{3}\right] \\
\text { (i) Bond lengths }\end{array}$} \\
\hline $\mathrm{N}-\mathrm{P}(1)$ & $1.574(3)$ & $C(32 A)-C(33 A)$ & $1.375(6)$ \\
\hline$N-P(2)$ & $1.575(3)$ & $C(33 A)-C(34 A)$ & $1.388(8)$ \\
\hline$P(1)-C(11 A)$ & $1.799(4)$ & $C(34 A)-C(35 A)$ & $1.359(9)$ \\
\hline$P(1)-C(21 A)$ & $1.796(4)$ & $C(35 A)-C(36 A)$ & $1.394(7)$ \\
\hline$P(1)-C(31 A)$ & $1.808(4)$ & $C(11 B)-C(12 B)$ & $1.376(5)$ \\
\hline$P(2)-C(11 B)$ & $1.804(4)$ & $C(11 B)-C(16 B)$ & $1.383(5)$ \\
\hline$P(2)-C(21 B)$ & $1.794(4)$ & $C(12 B)-C(13 B)$ & $1.403(6)$ \\
\hline$P(2)-C(31 B)$ & $1.803(4)$ & $C(13 B)-C(14 B)$ & $1.367(8)$ \\
\hline \multirow[t]{2}{*}{$C(11 A)-C(12 A)$} & $1.386(6)$ & $C(14 B)-C(15 B)$ & $1.372(7)$ \\
\hline & & $C(15 B)-C(16 B)$ & $1.384(6)$ \\
\hline$C(11 A)-C(16 A)$ & $1.403(6)$ & $C(21 B)-C(22 B)$ & $1.386(6)$ \\
\hline$C(12 A)-C(13 A)$ & $1.374(6)$ & $C(21 B)-C(26 B)$ & $1.402(5)$ \\
\hline$C(13 A)-C(14 A)$ & $1.353(7)$ & $\mathrm{C}(22 \mathrm{~B})-\mathrm{C}(23 \mathrm{~B})$ & $1.370(6)$ \\
\hline$C(14 A)-C(15 A)$ & $1.367(7)$ & $C(23 B)-C(24 B)$ & $1.373(7)$ \\
\hline$C(15 A)-C(16 A)$ & $1.375(6)$ & $C(24 B)-C(25 B)$ & $1.373(7)$ \\
\hline
\end{tabular}


TABLE 3 (continued)

\begin{tabular}{|c|c|c|c|}
\hline$\overline{C(21 A)-C(22 A)}$ & $1.382(5)$ & $(C(25 B)-C(26 B)$ & $1.376(6)$ \\
\hline$C(21 A)-C(26 A)$ & $1.404(5)$ & $C(3 \mid B)-C(.2 B)$ & $1.392(6)$ \\
\hline$C(22 A)-C(23 A)$ & $1.384(6)$ & $(C(3 \mid B)-C(36 B)$ & $1.3 \times 165)$ \\
\hline$C(23 A)-C(24 A)$ & $1.389(7)$ & $(C(32 B)-C(33 B)$ & $1.364(6)$ \\
\hline$C(24 A)-C(25 A)$ & $1.387(7)$ & $(C(33 B)-(84 B)$ & $1.372(7)$ \\
\hline$C(25 A)-C(26 A)$ & $1.372(6)$ & $C(34 B)-(15 B)$ & $1.350(7)$ \\
\hline$C(31 A)-C(32 A)$ & $1.391(6)$ & $(C(3 . \bar{B})-(C, 36 B)$ & $1.240(6)$ \\
\hline \multirow[t]{2}{*}{$(31 \mathrm{~A})-(\mathrm{C}, 3 \mathrm{~A})$} & $1.381(0)$ & & \\
\hline & $n=1$ & $n=2$ & $n=-3$ \\
\hline Mo- $C(n)$ & $1.917(4)$ & $1.924(4)$ & $1.926(4)$ \\
\hline $\mathrm{Mo}-\mathrm{N}(n \mathrm{l})$ & $2.288(3)$ & $2.274(3)$ & $2.279(3)$ \\
\hline $\mathrm{B}-\mathrm{N}(n 2)$ & $1.530(6)$ & $1.535(6)$ & $1545(6)$ \\
\hline$C(n)-O(n)$ & $1.167(5)$ & $1.176(5)$ & 1.1025, \\
\hline $\mathrm{N}(n 1)-\mathrm{N}(n 2)$ & $1.364(4)$ & $1.359(4)$ & $(.300)(5)$ \\
\hline $\mathrm{N}(n 2)-\mathrm{C}(n 3)$ & $1.340(5)$ & $1.3 .32(5)$ & $1.331(0)$ \\
\hline $\mathrm{C}(n 3)-\mathrm{N}(n 4)$ & $1.311(5)$ & $1.305(6)$ & $1.322(7)$ \\
\hline $\mathrm{N}(n 4)-\mathrm{C}\left(n_{5}\right)$ & $1.349(5)$ & $1 . .340(6)$ & $1.28+6(7)$ \\
\hline$C(n 5)-N(n 1)$ & $1.32065)$ & $1.318(5)$ & $131166)$ \\
\hline \multicolumn{4}{|l|}{ (ii) Bond angles } \\
\hline $\mathrm{C}(\mathrm{l})-\mathrm{Mo}-\mathrm{C}(2)$ & $83.84(18)$ & $C(1)-M o N(11)$ & $173.13(15)$ \\
\hline $\mathrm{C}(1)-\mathrm{Mo}-\mathrm{C}(3)$ & $90.37(18)$ & $C(1)-M O-N(21)$ & $95.07(10)$ \\
\hline $\mathrm{C}(2)-\mathrm{Mo}-\mathrm{C}(3)$ & $84.35(18)$ & $(2(1)-M n-N(31)$ & $07(1.4(1.5)$ \\
\hline $\mathrm{N}(11)-\mathrm{Mo}-\mathrm{N}(2 \mathrm{l})$ & $79.93(11)$ & $((2)-M o-N(11)$ & $101.1 .7(14)$ \\
\hline$N(11)-M o-N(31)$ & $77.61(11)$ & $(\mathrm{C})-\mathrm{Mo}-\mathrm{N}(2))$ & $178.91(14)$ \\
\hline$N(21)-M o-N(31)$ & $81.1+(12)$ & $C(2)-M o N(31)$ & $40.07(15)$ \\
\hline$N(12)-B-N(22)$ & $107.9(3)$ & $((3)-M O-N(11)$ & $94.79(1.5)$ \\
\hline$N(12)-B-N(32)$ & $107.1(3)$ & (C)-Mo-N(21) & 95.580141 \\
\hline$N(22)-B-N(32)$ & $108.8(3)$ & $\left(3^{3}\right)-M 0-N(31)$ & $172.11(15)$ \\
\hline$N-P(2)-C(1 \mid B)$ & $108.56(17)$ & $P(1)((31 /)-C(36 A)$ & $119.9(3)$ \\
\hline $\mathrm{N}-\mathrm{P}(2)-\mathrm{C}(2 \mid \mathrm{B})$ & $116.17(17)$ & $(8(32 A)-((31 A)-C(36 A)$ & $119.7(4)$ \\
\hline $\mathrm{N}-\mathrm{P}(2)-\mathrm{C}(3 \mid \mathrm{B})$ & $112.01(18)$ & $C(3 \mid A)-(C 32 A)-C(33 A)$ & $12(1.2(4)$ \\
\hline$C(11 B)-P(2)-C(21 B)$ & $106.74(18)$ & $(2(32 A)-C(33 A)(C(3+N)$ & $119.9(5)$ \\
\hline$(C(11 B)-P(2)-C(31 B)$ & $104.77(17)$ & $((33 A)-(C 3 A)-C(35 A)$ & $120.4(4)$ \\
\hline$C(21 B)-P(2)-C(31 B)$ & $107.88(18)$ & $(C 34 A)-(635)-(36 A)$ & $120.2(5)$ \\
\hline$P(1)-C(11 A)-C(12 A)$ & $121.2(3)$ & $((31 A)(36 A)-(35 A)$ & $119.9(4)$ \\
\hline$P(1)-C(11 A)-C(16 A)$ & $119.2(3)$ & $P(2)(C(1 \mid B)((12 B)$ & $119.4(3)$ \\
\hline$C(12 A)-C(11 A)-C(16 A)$ & $119.3(4)$ & $P(2) C(113)-C(16 B)$ & $120.0(3)$ \\
\hline$C(11 A)-C(12 A)-C(13 A)$ & $119.6(4)$ & $C(12 B)-C(11 B)-C(16 B)$ & $120.5(4)$ \\
\hline$C(12 A)-C(13 A)-C(14 A)$ & $120.8(4)$ & $C(11 B)-(C 12 B)-((13 B)$ & $119,64+$ \\
\hline$C(13 A)-C(14 A)-C(15 A)$ & $120.6(4)$ & $((12 B)-C(13 B)-C(14 B)$ & $110.9(4)$ \\
\hline$C(14 A)-C(15 A)-C(16 A)$ & $120.5(4)$ & $((13 B)-(14 B) C(15 B)$ & $120.8(4)$ \\
\hline$(C(11 A)-C(16 A)-C(15 A)$ & $119.1(4)$ & $(C(14 B)-C(15 B)-C(16 B)$ & $119.8(4)$ \\
\hline$P(1)-C(21 A)-C(22 A)$ & $122.4(3)$ & $(C(11 B)-C(16 B)-C(15 B)$ & $199.8(4)$ \\
\hline$P(1)-C(21 A)-C(26 A)$ & $117.4(3)$ & $P(2)-C(21 B)-C(22 B)$ & $120.2(3)$ \\
\hline$(C(22 A)-C(21 A)-C(26 A)$ & $120.1(3)$ & $P(2)-((21 B)-(20 B)$ & $120.7(3)$ \\
\hline$(\alpha 21 A)-C(22 A)-(23 A)$ & $119.5(4)$ & $((22 B) C(21 B)-C(26 B)$ & 119.1131 \\
\hline$C(22 A)-C(23 A)-C(24 A)$ & $120.9(4)$ & $C(21 B)-((22 B)-(C 23 B)$ & $120.6(4)$ \\
\hline$P(1)-N-P(2)$ & $141.19(21)$ & $C(22 B)(C 23 B)(C 2 A B)$ & $120.1(1)$ \\
\hline$N-P(1)-C(11 A)$ & $114.31(17)$ & $C(23 B)-C(24 B)(C 2 B B)$ & $120.2(4)$ \\
\hline$N-P(1)-C(21 A)$ & $109.42(17)$ & $C(24 B)-C(25 B)-(C 26 B)$ & $120.6(4)$ \\
\hline $\mathrm{N}-\mathrm{P}(1)-\mathrm{C}(31 \mathrm{~A})$ & $110.14(18)$ & $(C 2 B B)-C(26 B)-(C 2 B B)$ & $119.4(4)$ \\
\hline$C(11 A)-P(1)-C(21 A)$ & $109.77(18)$ & $P(2) \cdots((31 B)-C(32 B)$ & $121.0 \times 33$ \\
\hline$C(11 A)-P(1)-C(31 \Lambda)$ & $107.06(17)$ & $P(2)-C(3 \mid B)-((36 B)$ & $110.3(3)$ \\
\hline$C(21 A)-P(1)-C(31 A)$ & $105.77(18)$ & $C(32 B)-C(31 B)-(136 B)$ & $119.4(4)$ \\
\hline$C(23 A)-C(24 A)-C(25 A)$ & $118.8(4)$ & $C(3 \mid B)-C(32 B)-((3.3 B)$ & $120.1(4)$ \\
\hline$C(24 A) C(25 A) C(26 A)$ & $121.2(4)$ & $(C(32 B)-C(33 B)((34 B)$ & $120.6(4)$ \\
\hline$C(21 A)-C(26 A)-C(25 A)$ & $119.3(4)$ & $C(33 B)-C(34 B)-(C(35 B)$ & $120.0(4)$ \\
\hline \multirow[t]{2}{*}{$P(1)-C(31 A)-C(32 A)$} & $120.6(3)$ & $C(34 B)-C(35 B)-C(36 B)$ & $120,9(4)$ \\
\hline & & $C(31 B)-C(36 B)-C(3513)$ & $118,9(4)$ \\
\hline
\end{tabular}


TABLE 3 (continued)

\begin{tabular}{llll}
\hline & $n=1$ & $n=2$ & $n=3$ \\
\cline { 2 - 3 } Mo-N $(n 1)-\mathrm{N}(n 2)$ & $122.29(23)$ & $121.35(22)$ & $121.36(23)$ \\
$\mathrm{Mo}-\mathrm{N}(n 1)-\mathrm{C}(n 5)$ & $133.9(3)$ & $134.8(3)$ & $134.9(3)$ \\
$\mathrm{Mo}-\mathrm{C}(n)-\mathrm{O}(n)$ & $178.9(4)$ & $174.6(3)$ & $177.6(4)$ \\
$\mathrm{B}-\mathrm{N}(n 2)-\mathrm{N}(n 1)$ & $121.1(3)$ & $121.7(3)$ & $121.6(3)$ \\
$\mathrm{B}-\mathrm{N}(n 2)-\mathrm{C}(n 3)$ & $131.8(3)$ & $131.2(3)$ & $130.7(4)$ \\
$\mathrm{N}(n 1)-\mathrm{N}(n 2)-\mathrm{C}(n 3)$ & $107.0(3)$ & $107.0(3)$ & $107.4(4)$ \\
$\mathrm{N}(n 2)-\mathrm{C}(n 3)-\mathrm{N}(n 4)$ & $112.9(4)$ & $112.5(3)$ & $112.3(4)$ \\
$\mathrm{C}(n 3)-\mathrm{N}(n 4)-\mathrm{C}(n 5)$ & $101.9(4)$ & $102.3(3)$ & $101.7(4)$ \\
$\mathrm{N}(n 4)-\mathrm{C}(n 5)-\mathrm{N}(n 1)$ & $114.4(4)$ & $114.4(4)$ & $115.0(5)$ \\
$\mathrm{N}(n 2)-\mathrm{N}(n 1)-\mathrm{C}(n 5)$ & $103.7(3)$ & $103.8(3)$ & $103.7(4)$ \\
\hline
\end{tabular}

using $\psi$ scans. Related crystal data (Table 1), final coordinates of the non-hydrogen atoms (Table 2), and selected bond lengths and bond angles (Table 3 ) are reported. The anisotropic displacement coefficients of the atoms, the $\mathrm{H}$-atom coordinates and structural factors are available from the authors. The ORTEP plots for anions, $\left[\mathrm{Mo}\left(\mathrm{HBPz}_{3}\right)(\mathrm{CO})_{3}\right]^{-}$and $\left[\mathrm{Mo}\left(\mathrm{HBTz}_{3}\right)_{-}\right.$ $\left.(\mathrm{CO})_{3}\right]^{-}$were all quite similar and are shown as one plot (Fig. 1).

\section{Results and discussion}

The $\mathrm{KHBTz}_{3}$ salt can be obtained in an analogous way to the preparation of $\mathrm{KHBP}_{3}$ [12] from the melt reaction of $\mathrm{KBH}_{4}$ in excess $\mathrm{HTz}$ at $200^{\circ} \mathrm{C}$. Although a

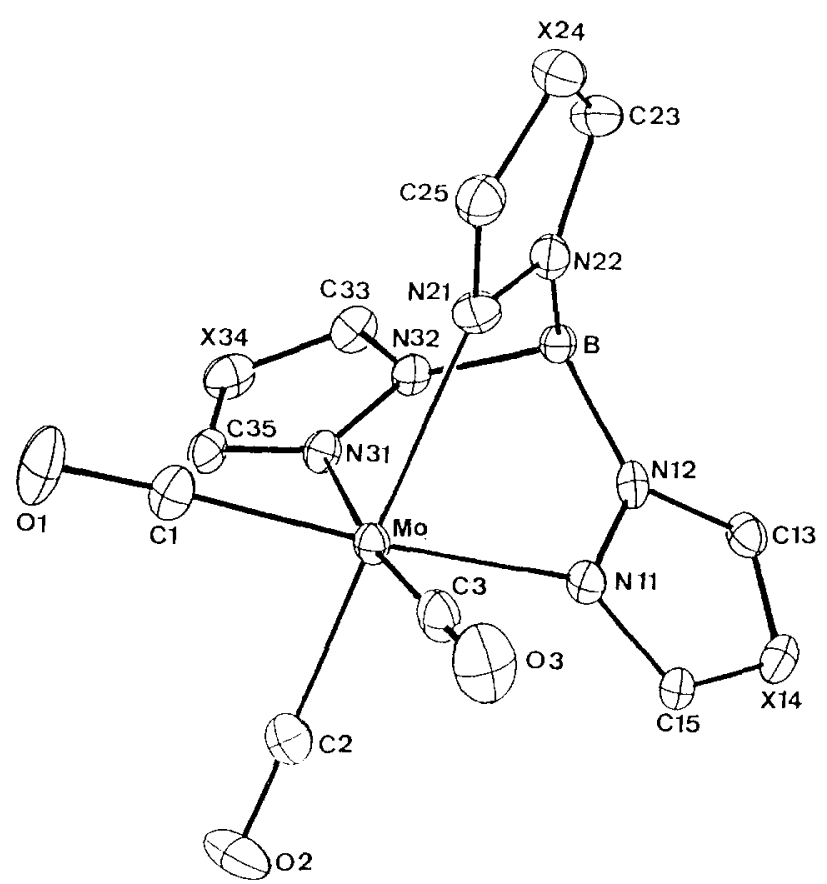

Fig. 1. ORTEP plot for anions, $\left[\mathrm{Mo}\left(\mathrm{HBPz}_{3}\right)(\mathrm{CO})_{3}\right]^{-}(\mathrm{X}=\mathrm{C})$ and $\left[\mathrm{Mo}\left(\mathrm{HBTz}_{3}\right)(\mathrm{CO})_{3}\right]^{-}(\mathrm{X}=\mathrm{N})$, showing the numbering scheme. The ellipsoids were drawn at the $50 \%$ probability level. similar reaction between $\mathrm{NaBPh}_{4}$ and $\mathrm{HPz}$ at high temperatures gives only the disubstituted product $\mathrm{NaPh}_{2} \mathrm{BPz}_{2}$ [13], the reaction between $\mathrm{NaBPh}_{4}$ and HTz first affords $\mathrm{NaPh}_{2} \mathrm{BTz}_{2}[14]$ and then $\mathrm{NaPhBTz}_{3}$, reflecting the proton of $\mathrm{HTz}$ being more acidic than that of $\mathrm{HPz}$ [15]. The $\mathrm{L}$ anion $\left(\mathrm{L}^{-}=\mathrm{HBTz}_{3}{ }^{-}\right.$or $\mathrm{PhBTz}_{3}{ }^{-}$) can replace the three mutually cis-carbonyls of $\left[\mathrm{Mo}(\mathrm{CO})_{6}\right]$ in a similar way to $\mathrm{HBPz}_{3}{ }^{-}, \mathrm{HBPz}_{3}^{\prime-}$ [17], $\mathrm{HBPz}_{3}^{\prime \prime}-$ [8], $\mathrm{HBBz}_{3}{ }^{-}$[6a], or $\mathrm{HBPz}_{3}^{\star-}$ [16] $\left(\mathrm{Pz}^{\prime}=\right.$ 3,5-dimethylpyrazol-1-yl; $\mathrm{Pz}^{\prime \prime}=3,4,5$-trimethylpyrazol1-yl; $\quad \mathrm{Pz}^{\star}=3$-methylpyrazol-1-yl; $\quad \mathrm{Bz}=$ benzotriazol-1yl) to give $\left[\mathrm{MoL}(\mathrm{CO})_{3}\right]^{-}$. The addition of electrophiles to $\mathrm{M}\left[\mathrm{MoL}(\mathrm{CO})_{3}\right](\mathrm{M}=\mathrm{Na}$ or $\mathrm{K})$ or $\left[\mathrm{Et}_{4} \mathrm{~N}\right]\left[\mathrm{MoL}(\mathrm{CO})_{3}\right]$ converts the salts readily into $\left[\mathrm{MoL}(\mathrm{CO})_{3} \mathrm{X}\right](\mathrm{X}=\mathrm{Br}$ or 1$),\left[\mathrm{MoL}(\mathrm{CO})_{2}(\mathrm{NO})\right]$, and $\left[\mathrm{MoL}(\mathrm{CO})_{2}\left(\eta^{3}\right.\right.$-allyl $\left.)\right]$ (Scheme 1). Apparently, the reaction chemistry of $\left[\mathrm{Mo}\left(\mathrm{HBTz}_{3}\right)(\mathrm{CO})_{3}\right]^{-}$or $\left[\mathrm{Mo}\left(\mathrm{PhBTz}_{3}\right)(\mathrm{CO})_{3}\right]^{-}$is quite similar to that of $\left[\mathrm{Mo}\left(\mathrm{HBPz}_{3}\right)(\mathrm{CO})_{3}\right]^{-},\left[\mathrm{Mo}\left(\mathrm{HBPz}_{3}^{\prime}\right)\right.$ $\left.(\mathrm{CO})_{3}\right]^{-}[1]$ or $\left[\mathrm{Mo}\left(\mathrm{HBBz}_{3}\right)(\mathrm{CO})_{3}\right]^{-}$[6a] towards the electrophiles we used in this study.

All the anions, $\left[\mathrm{MoL}^{\prime}(\mathrm{CO})_{3}\right]^{-} \quad\left(\mathrm{L}^{\prime}=\mathrm{HBPz}_{3}{ }^{-}\right.$, $\mathrm{HBPz}_{3}^{\prime-}, \mathrm{HBPz}_{3}^{\prime \prime-}, \mathrm{HBPz}_{3}^{*-}, \mathrm{HBBz}_{3}^{-}, \mathrm{HBTz}_{3}{ }^{-}$, or $\mathrm{PhBTz}_{3}{ }^{-} ; \mathrm{Pz}^{\prime \prime}=3,4,5$-trimethylpyrazol-1-yl), belong to the point group of $\mathrm{C}_{3 \mathrm{v}}$ and display one strong $\left(\mathrm{A}_{1}\right)$ and one very strong $(E)$ carbonyl stretching bands in solution. The averaged $\nu(\mathrm{CO})$ values taken as $1 / 3 \nu\left(\mathrm{A}_{1}\right)+$ $2 / 3 \nu(\mathrm{E})$ for the anions measured in $\mathrm{MeCN}$ are calculated as follows: $\mathrm{L}^{\prime}=\mathrm{HBBz}_{3}{ }^{-}, 1834 \mathrm{~cm}^{-1}$ [6a]; $\mathrm{HBPz}_{3}{ }^{-}, 1806 \mathrm{~cm}^{-1} ; \mathrm{HBPz}_{3}^{\prime-}, 1798 \mathrm{~cm}^{-1}[17] ; \mathrm{HBPz}_{3}^{\prime \prime}$,

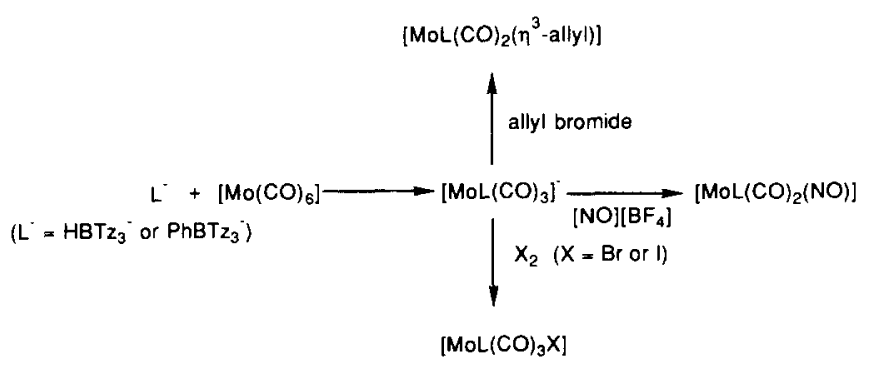

Scheme 1. 
$1793 \mathrm{~cm}^{-1}[8] ; \mathrm{HBPz}_{3}^{\star \cdots}, 1799 \mathrm{~cm}^{-1}$ [16]; HBTz $z_{3} \cdots, 1819$ $\mathrm{cm}^{-1}$; and $\mathrm{PhBTz}_{3}^{--}, 1818 \mathrm{~cm}^{-1}$, indicating the increasing order of electron donating power as $\mathrm{HBBz}_{3}$ $<\mathrm{HBTz}_{3}{ }^{-}, \mathrm{PhBTz}_{3}{ }^{\cdots}<\mathrm{HBPz}_{3}{ }^{-}<\mathrm{HBPz}_{3}^{\prime} . \mathrm{HBPz}_{3}^{\star-\cdots}<$ $\mathrm{HBPz}_{3}^{\prime \prime-}$. The weaker donating ability of $\mathrm{HBTz}_{3}$ "compared with that of $\mathrm{HBPz}_{3}$ is compatible with $\mathrm{HTz}$

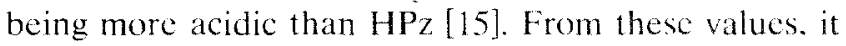
is clear that (1) the replacement of the hydrogen atom on the boron atom by a phenyl group in $\mathrm{HBTz}_{3}$ " to give $\mathrm{PhBTz}_{3}{ }^{-}$) or the replacement of the hydrogen atoms on the pyrazolyl ring-5 position with the methyl groups in $\mathrm{HBPz}_{3}^{*}$ (to give $\mathrm{HBP}_{3}^{\prime}$ ) does not change at all the donating ability of the relevant ligands; (2) the substitution of the hydrogen atoms on pyrazolyl ring $-3,-4$, or -5 position with methyl groups. converting from $\mathrm{HBP}_{3}$ " to $\mathrm{HBPz}_{3}^{\prime--}$ or $\mathrm{HBPz}_{3}^{\prime--}$ increases the ability slightly and (3) the replacement of the $\mathrm{CH}$ atoms on pyrazolyl ring-3 or -4 position by a nitrogen atom in making $\mathrm{Bz}$ or $\mathrm{T} z$, respectively, do weaken this ability. As reported earlier [6a], the complete reaction between $\left[\mathrm{Mo}\left(\mathrm{HBBz}_{3}\right)(\mathrm{CO})_{3}\right]^{-}$and allyl bromide needs about $17 \mathrm{~h}$ while a similar reaction takes only about $3 \mathrm{~h}$ for $\left[\mathrm{Mo}\left(\mathrm{HBTz}_{3}\right)(\mathrm{CO})_{3}\right]^{-}$or $\left[\mathrm{Mo}\left(\mathrm{PhBT}_{3}\right)(\mathrm{CO})_{3}\right]^{\cdots}$, showing that the much weaker donativity of $\mathrm{HBBz}_{3}$ retards more strongly the oxidative allyl bromination of $\left[\mathrm{MoL}(\mathrm{CO})_{3}\right]^{\circ} \quad\left(\mathrm{L}^{-}=\mathrm{HBTz}_{3}{ }^{-}, \mathrm{PhBT}_{3}{ }^{-}\right.$or $\left.\mathrm{HBBz}_{3}{ }^{-}\right)$ than $\mathrm{HBT}_{z_{3}}$ or PhBTz $z_{3}$. Thus, the replacement of the $\mathrm{CH}$ atoms on the ring-3 position by a nitrogen atom has a stronger effect than the substitution on the ring-4 position.

In order further to compare the relevant structure features of $\left[\mathrm{MoL}^{\prime}(\mathrm{CO})_{3}\right] \quad\left(\mathrm{L}^{\prime}=\mathrm{HBP}_{2}, \cdots, \mathrm{HBPz}_{3}^{\prime}\right.$, $\mathrm{HBT}_{3}{ }^{-}$, or $\mathrm{HBPz}_{3}^{*}$ ), we determined the solid-state structures of $\left[\mathrm{NEt}_{4}\right]\left[\mathrm{Mo}\left(\mathrm{HBPz}_{3}\right)(\mathrm{CO})_{3}\right]$ (1) and $\left[\mathrm{N}\left(\mathrm{PPh}_{3}\right)_{2}\right]\left[\mathrm{Mo}\left(\mathrm{HBTz}_{3}\right)(\mathrm{CO})_{3}\right](4)$. The two anions are isostructural (Fig. 1), as expected, to $\left[\mathrm{NEt}_{4}\right][\mathrm{Mo}-$
$\left.\left(\mathrm{HBPZ}_{3}^{\prime}\right)(\mathrm{CO})_{3}\right](3)[19]$ and $\left[\mathrm{NEt}_{4}\right]\left[\mathrm{Mo}\left(\mathrm{HBPz}_{3}^{*}\right)(\mathrm{CO})_{3}\right]$ (2) [16]. To our surprise. 1 and 4 are even X-ray isomorphous in a space group of Pbca. Apparently, the cone angles of $\mathrm{HBPz}_{3}$ and $\mathrm{HBTz}_{3}$ should be similar and the two similar anions $\left[\mathrm{Mo}\left(\mathrm{HBPz}_{3}\right)(\mathrm{CO})_{3}\right]^{-}$and $\left[\mathrm{Mo}\left(\mathrm{HBT}_{3}\right)(\mathrm{CO})_{3}\right]^{-}$being larger than the cations $\mathrm{NEl}_{4}$. or $\mathrm{N}\left(\mathrm{PPh}_{3}\right)_{2}$. determines the packing habit in the crystal [18]. As the methyl groups replace the hydrogen atoms symmetrically at the pyrazolyl ring- 3 and -5 positions, converting $\mathrm{HBPz}_{;}$into $\mathrm{HBPz}_{;}^{\prime}$, this changes the habit to the space group of lower symmetry $\left(\right.$ Pla $\left._{2}\right)$ for $\left[\mathrm{NE}_{4}\right]\left[\mathrm{Mo}\left(\mathrm{HBPz}_{3}\right)(\mathrm{CO})_{3}\right][19]$ and the less symmetric replacement of the hydrogen atoms, at the ring-3 position only, by methyl groups in the process of formation of $\mathrm{HBPz}_{3}^{*}$ from $\mathrm{HBPz}_{3}$, results in the space group of much lower symmetry $\left(P_{2}\right)$ for $\left[\mathrm{NEt}_{4}\right]\left[\mathrm{Mo} \mathrm{HBP}_{3}^{*}\right)\left(\mathrm{CO}_{3}\right][16]$.

Some averaged bond lengths and angles calculated from the structures of anions $\left[\mathrm{MOL}^{\prime}(\mathrm{CO})_{3}\right]\left(\mathrm{L}^{\prime}=\right.$ $\mathrm{HBPz}_{3}, \mathrm{HBPz}_{;}, \mathrm{HBTz}_{3}$, or $\mathrm{HBPz}_{3}^{*}$, are listed in Table 4. From this table, it is clear that the angle $C(n)-$ Mo- $C\left(n^{\prime}\right)$ of $84.4^{\circ}$ in 3 is smaller than that of $88.0^{\circ}$ in 1 . reflecting the different steric encumbrances (the cone angle is measured as 180 or $184^{\circ}$ for $\mathrm{HBPz}$; and 224 or $225^{\circ}$ for $\mathrm{HBPZ}_{3}$ [20,21]). Since the angle. $\mathrm{C}(\mathrm{n})-\mathrm{Mo}-\mathrm{C}\left(\mathrm{n}^{\prime}\right)$, is $85.3^{\circ}$ in 2 (Table 4 ), it is reasonable to assume that the cone angle of $\mathrm{HBP}_{3}^{*}$ is less than that of $\mathrm{HBPz}_{3}$ though larger than in $\mathrm{HBP}_{3}$. How ever. the angle should be used with caution as the Mo-N(nl) and Mo-C(n) distances may be different. The angle of $86.2^{\circ}$ in 4 , larger than that in 1 , cannot be used to infer that the cone angle for $\mathrm{HBTz}_{3}$ is larger than that for $\mathrm{HBPz}_{3}$, rather it has something to do with the longer $\mathrm{Mo}-\mathrm{N}$ distance $(\mathrm{Mo}-\mathrm{N}=2.280 \AA \mathrm{A}$ in 4 (s. $2.265 \AA$ in 1 and $\mathrm{Mo}-\mathrm{C}=1.922 \AA$ in $4 \mathrm{rs} .1 .925 \AA$ in 1). which may reflect the donativity of $\mathrm{HBtz}_{3}$ - being

TABLE 4. Averaged bond lengths $(\hat{A})$ and angles () for $\left[\mathrm{MoL}(C O)_{3}\right]$

\begin{tabular}{|c|c|c|c|c|}
\hline $\mathrm{L} \cdots$ & $\mathrm{HBPz}_{3}$ & $\mathrm{HBPz}^{\star}$ & $\mathrm{HBP}_{7}$ & $\mathrm{HBTz}_{3}$ \\
\hline$M o-C(n)$ & $1.925[1]^{:}$ & $1.928[10]$ & $1.941[4]$ & $1.922[5]$ \\
\hline$C(n)-O(n)$ & $1.176[9]$ & $1.168[14]$ & $1.167[5]$ & $1.168[7]$ \\
\hline $\mathrm{Mo}-\mathrm{N}(n 1)$ & $2.265[6]$ & $2.285[5]$ & $2.263[5]$ & $2.280[7]$ \\
\hline $\mathrm{B}-\mathrm{N}(n 2)$ & $1.538[12]$ & $1.532[8]$ & $1.535[11]$ & $1.537[8]$ \\
\hline $\mathrm{N}(n 1)-\mathrm{N}(n 2)$ & $1.366[6]$ & $1.363[5]$ & $1.376[11]$ & $1.361[4]$ \\
\hline$N(n 2)-C(n 3)$ & $1.348[7]$ & $1.346[17]$ & $1.365[9]$ & $1.29+[5]$ \\
\hline $\mathrm{C}(n 3)-\mathrm{X}(n 4)^{\mathrm{b}}$ & $1,36 \%[5]$ & $1.350[20]$ & $1.351[16]$ & 1.313191 \\
\hline$X(n 4)-C(n 5)$ & $1.378[7]$ & $1.391[1.5]$ & $1.788[27]$ & $1.3+7[2]$ \\
\hline$C(n 5)-N(n 1)$ & $1.336[10]$ & $1 . .335 \mid 67$ & $1 . .341[29]$ & $1.316[5]$ \\
\hline $\mathrm{N}(n 1)-\mathrm{Mo}-\mathrm{N}\left(n^{\prime} 1\right)$ & $80.4[5]$ & $81.11[8]$ & $31.3\lfloor 11 !$ & $79.6[18]$ \\
\hline $\mathrm{N}\left(n^{2}\right)-\mathrm{B}-\mathrm{N}\left(n^{\prime} 2\right)$ & $109.1[2]$ & $109.0[7]$ & $109.2\{3\}$ & $107.9[9]$ \\
\hline$C(n)-M_{0}-C\left(n^{\prime}\right)$ & $88.0[29]$ & $85.3[10]$ & $84.4[11]$ & $80.2[36]$ \\
\hline Reference & this work & {$[16]$} & {$[19]$} & this work \\
\hline
\end{tabular}

"Standard deviations in square brackets were calculated by $\sigma=(\Sigma(x-\bar{x}) /(N-1)+N=$ the number of data used; $x=C$ for anions with $L^{\prime}=\mathrm{HBPZ}_{3}, \mathrm{HBPZ}_{3}^{*--}$ or $\mathrm{HBPz}_{3}^{\prime}$ and $\mathrm{X}=\mathrm{N}$ for for $\mathrm{HBTz}$ 
weaker than that of $\mathrm{HBPz}_{3}{ }^{-}$, as observed in the larger averaged carbonyl-stretching value stated above. The Mo-N distance is $2.285 \AA$ in 2 but $2.263 \AA$ in 3 , giving only weak support to the 5-methyl-group effect for nickel pseudohalides suggested by Trofimenko et al. [9], whereby the presumed nonbonded repulsive interactions among the methyl groups at the ring-5 position are alleviated by tightening of the "bite" of the tridentate ligands around the metal atom with shortening Mo-N distances. It is probably true from the similarity of the Mo-N distances of $2.265 \AA$ in 1 and $2.263 \AA$ in 3 that the averaged value of $2.264 \AA$ from the two may represent the closest distance between the tripodal ligand and the metal atom in 1-4. Thus, if the 5methyl-group effect is present at all in metal compounds, this effect should be manifested more clearly in the four-coordinate complexes such as the nickel compounds used by these other workers [9] compared with that in the six-coordinate compounds used in this paper.

\section{Acknowledgment}

We wish to thank the National Science Council of the Republic of China for financial support of this research (Contract No. NSC82-0208-M006-32).

\section{References}

1 (a) S. Trofimenko, Acc. Chem. Res., 4 (1971) 17; (b) S. Trofimenko, Chem. Rev., 72 (1972) 497; (c) S. Trofimenko, Adv. Chem., 150 (1976) 289; (d) A. Shaver, J. Organomet. Chem. Libr., 3 (1977) 157; (e) S. Trofimenko, Prog. Inorg. Chem., 34 (1986) 115.

2 (a) D.G. Hamilton, X.-L. Lou and R.H. Crabtree, Inorg. Chem., 28 (1989) 3198; (b) R.S. Tanke and R.H. Crabtree, Inorg. Chem., 28 (1989) 3444.

3 (a) N. Kitajima, K. Fujisawa, Y. Moro-oka and K. Toriumi, $J$. Am. Chem. Soc., 111 (1989) 8975; (b) R. Han and G. Parkin, J.
Am. Chem. Soc., 112 (1990) 3662; (c) N. Kitajima, T. Koda, Y. Iwata and Y. Moro-oka, J. Am. Chem. Soc., 112 (1990) 8833; (d) I.A. Degam, W.A. Herrmann and E. Herdtweck, Chem. Ber., 123 (1990) 1347.

4 I.W. Fagan, Jr., B.S. Hagerty, A.I. Rheingnld, S.C. Sendlinger and K.H. Theopold, J. Am. Chem. Soc., 112 (1990) 2445.

5 (a) C.K. Ghosh and W.A.G. Graham, J. Am. Chem. Soc., 109 (1987) 4726; (b) C.K. Ghosh, P.S. Rodgers and W.A.G. Graham, J. Chem. Soc., Chem. Commun., (1988) 1511; (c) C.K. Ghosh and W.A.G. Graham, J. Am. Chem. Soc., 111 (1989) 375; (d) C.K. Ghosh, J.K. Hoyano, R. Krentz and W.A.G. Graham, J. Am. Chem. Soc., 111 (1989) 5480; (e) F.A. Cotton and R.L. Luck, Inorg. Chem., 28 (1989) 3210.

6 (a) K.-B. Shiu, F.-M. Shen, S.-L. Wang and S.-C. Wei, J. Organomet. Chem., 372 (1989) 251; (b) F.J. Lalor, S. Miller and N. Garvey, J. Organomet. Chem., 356 (1988) C57.

7 (a) M.D. Curtis and K.-B. Shiu, Inorg. Chem., 24 (1985) 1213; (b) M.D. Curtis, K.-B. Shiu and W.M. Butler, J. Am. Chem., Soc., 108 (1986) 1550; (c) M.D. Curtis, K.-B. Shiu, W.M. Butler and J.C. Huffman, J. Am. Chem. Soc., 108 (1986) 3335.

8 K.-B. Shiu and L.-Y. Lee, J. Chin. Chem. Soc., 36 (1989) 31.

9 S. Trofimenko, J.C. Calabrese, J.K. Kochi, S. Wolowiec, F.B. Hulsbergen and J. Reedijk, Inorg. Chem., 31 (1992) 3943.

10 D.F. Shriver (Ed.), Manipulation of Air-Sensitive Compounds, McGraw-Hill, New York, 1969.

11 K.-B. Shiu, C.-J. Chang, Y. Wang and M.-C. Cheng, J. Chin. Chem. Soc. (Taipei), 36 (1989) 25.

12 S. Trofimenko, Inorg. Synth., 12 (1970) 99.

13 S. Trofimenko, J. Am. Chem. Soc., 89 (1967) 6288.

14 Jean Y. Lee, M. Sc. Thesis, National Cheng Kung University, 1990.

15 A.R. Katritzky and C.W. Rees, Compr. Heterocycl. Chem., 5 (1984) 50 .

16 T.J. Desmond, F.J. Lalor, G. Ferguson and M. Parvez, I. Organomet. Chem., 277 (1984) 91.

17 S. Trofimenko, J. Am. Chem. Soc., 91 (1969) 588.

18 (a) F. Basolu, Coord. Chern. Rec., 3 (1968) 213; (b) A. Gavezzotti, J. Am. Chem. Soc., 105 (1983) 5220; (c) D.M.P. Mingo and A.L. Rohl, Inorg. Chem., 30 (1991) 3769.

19 C.P. Marabella and J.H. Enemark, J. Organomet. Chem., 226 (1982) 57.

20 E. Frauendorfer and H. Brunner, J. Organomet. Chem., 240 (1982) 371 .

21 S. Trofimenko, J.C. Calabrese and J.S. Thompson, Inorg. Chem., 26 (1987) 1507. 